\title{
First principles study of point defects in titanium oxycarbide
}

\author{
H. M. Pinto ${ }^{\text {a }}$ J. Coutinho ${ }^{\text {b }}$, M. M. D. Ramos ${ }^{\mathrm{a}}$, F. Vaz ${ }^{\mathrm{a}}$, L. Marques ${ }^{\mathrm{a}, *}$ \\ ${ }^{a}$ University of Minho, Department of Physics, Campus de Gualtar, 4710-057 Braga, Portugal \\ ${ }^{b}$ University of Aveiro, Department of Physics, Campus Santiago, 3810-193 Aveiro, Portugal
}

\begin{abstract}
We have performed first principles density functional theory calculations to study the formation energy of point defects in $\mathrm{TiC}$, $\mathrm{TiO}$ and TiCO compounds. The formation energy of isolated vacancies were obtained for different equilibrium conditions. For binary compounds, we have also calculated the formation energy of antisite defects. It was found that the defect formation energies strongly depend on the chemical environment. Our results show that $\mathrm{C}$ vacancies are easily formed in $\mathrm{TiC}$ and $\mathrm{TiCO}$. For the $\mathrm{TiO}$ compound, $\mathrm{Ti}$ vacancies are highly probable to occur and $\mathrm{O}$ vacancies are also easily formed under titanium rich atmosphere.
\end{abstract}

Key words: Defect formation, Titanium oxide, Titanium carbide

\section{Introduction}

Titanium oxycarbide is a multifunctional material crystalizing in the rocksalt structure. Changing the oxygen/carbon ratio it is possible to tune its mechanical and electrical properties from the metallic-like, such has high electrical conductivity and ductility to insulating behavior and extreme hardness of typical covalent bonded materials. $[1,2,3]$ This variety of properties has attracted the attention to this material due to its potential for a wide range of high technological applications. These materials presents a high concentration of defects, specially vacancies, which play an important role in the material properties. $[5,4]$ Thus, it is important to investigate the formation of isolated vacancies and antisite point defects in titanium oxycarbide, which can be obtained at different chemical environment.

In this work we present a theoretical study of point defects in titanium oxycarbides using first principles calculations. The formation energy of vacancies and antisite defects for the binary $\mathrm{TiC}$ and $\mathrm{TiO}$ compounds and the formation energy of vacancies in the ternary compound $\mathrm{TiC}_{0.5} \mathrm{O}_{0.5}$ are calculated by ab initio methods.

\section{Method}

The calculations were performed within the density functional theory (DFT), using the (VASP) code [7, 8, 9], with local density approximation (LDA) for the exchange-correlation energy and projector augmented wave (PAW)

Email addresses: 1sam@fisica.uminho.pt (L. Marques)

Preprint submitted to Plasma Processes \& Polymers

February 3, 2009 
pseudopotentials [10], in supercells containing 64 atoms. Cut-off energy $\left(\mathrm{E}_{\text {cut }}\right)$ of $500 \mathrm{eV}$ was used for the plane-wave basis set expansion. The Brillouin zone (BZ) was sampled using the Monkhorst-Pack scheme [11] with a mesh of $7 \times 7 \times 7$ points in the reciprocal unit cell.

The formation energy of a defect in a binary compound like TiC is given by [12]:

$$
\Omega_{D}=E_{D}-n_{T i} \mu_{T i}-n_{C} \mu_{C}
$$

where $E_{D}$ is the total energy of a supercell containing $n_{T i}$ Ti atoms, $n_{C} \mathrm{C}$ atoms and one defect, $\mu_{T i}$ and $\mu_{C}$ are the chemical potentials of $\mathrm{Ti}$ and $\mathrm{C}$, respectively. This equation may be written in the form

$$
\Omega_{D}=E_{D}^{\prime}-\frac{1}{2}\left(n_{C}-n_{T i}\right) \Delta \mu
$$

where

$$
E_{D}^{\prime}=E_{D}-\frac{1}{2}\left(n_{C}+n_{T i}\right) \mu_{T i C}^{B}-\frac{1}{2}\left(n_{C}-n_{T i}\right)\left(\mu_{C}^{B}-\mu_{T i}^{B}\right)
$$

The superscript $B$ in the chemical potential indicates the corresponding bulk value.The difference $\Delta \mu$ is defined as

$$
\Delta \mu=\left(\mu_{C}-\mu_{T i}\right)-\left(\mu_{C}^{B}-\mu_{T i}^{B}\right)
$$

The chemical potential of Ti and $\mathrm{C}$ should not exceed their bulk chemical potential, otherwise the respective precipitate would be formed. Consequently, $\mu_{T i} \leqslant \mu_{T i}^{B}$ and $\mu_{C} \leqslant \mu_{C}^{B}$. At thermal equilibrium, the sum of the Ti and $\mathrm{C}$ chemical potentials is equal to the chemical potential of bulk TiC per pair of atoms; $\mu_{T i}+\mu_{C}=\mu_{T i C}^{B}$. Then, the difference in the chemical potentials of both elements is restricted by the following condition

$$
-\Delta H \leqslant \Delta \mu \leqslant \Delta H
$$

where $\Delta H$ is the heat of formation of TiC. This determines the growth conditions during the defect formation: $\Delta \mu=\Delta H$ corresponds to Ti-rich limit and $\Delta \mu=-\Delta H$ corresponds to C-rich limit. The bulk chemical potentials of Ti, C, O, $\mathrm{TiC}$ and $\mathrm{TiO}$ were calculated using VASP, assuming a hcp structure in case of titanium, the diamond structure for carbon and a $\mathrm{NACl}$ structure for $\mathrm{TiC}$ and $\mathrm{TiO}$ compounds. For the calculation of the chemical potential of oxygen we considered a large supercell with an oxygen molecule in the center.

In order to illustrate the application of the theoretical formalism described to ternary compounds, we will just consider in this work, as a reference, an ordered $\mathrm{TiCO}$ alloy with the composition $\mathrm{TiC}_{0.5} \mathrm{O}_{0.5}$ and the spatial arrangement illustrated in figure 1 for an eight atom supercell. In the case of a ternary compound, like TiCO, the defect formation energy is given by [13]

$$
\Omega_{D}=E_{D}-n_{T i} \mu_{T i}-n_{C} \mu_{C}-n_{O} \mu_{O}
$$

The Ti, $\mathrm{C}$ and $\mathrm{O}$ chemical potencials $\left(\mu_{T i}, \mu_{C}\right.$ e $\left.\mu_{O}\right)$ should not exceed their corresponding bulk values and the sum of those chemical potentials must satisfy the following conditions

$$
\mu_{T i}+\mu_{C} \leqslant \mu_{T i C}^{B}
$$




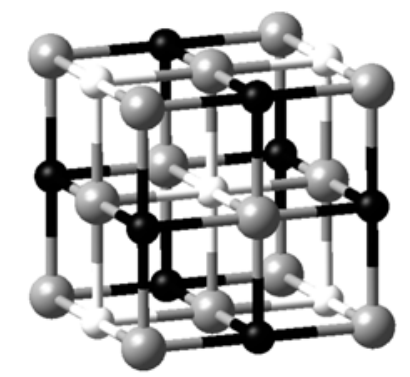

Figure 1: $\mathrm{NaCl}$ structure of $\mathrm{TiC}_{0.5} \mathrm{C}_{0.5}$. One sublattice is occupied by metal atoms (grey) and the other by carbon (black) and oxygen atoms (white), with the spatial distributions shown.

and

$$
\mu_{T i}+\mu_{O} \leqslant \mu_{T i O}^{B}
$$

At thermal equilibrium, the sum of the chemical potentials of the isolated elements is equal to to the chemical potential of the ternary compound,

$$
2 \mu_{T i}+\mu_{C}+\mu_{O} \leqslant \mu_{T i C_{0.5} O_{0.5}}^{B}
$$

This conditions limit the ranges over which the chemical potentials can vary. Defining two new parameters $\Delta \bar{\mu}$ and $\delta \bar{\mu}$

$$
\Delta \bar{\mu}=\left(\mu_{O}+\mu_{C}-2 \mu_{T i}\right)-\left(\mu_{O}^{B}+\mu_{C}^{B}-2 \mu_{T i}^{B}\right)
$$

and

$$
\delta \bar{\mu}=\left(\mu_{C}-\mu_{O}\right)-\left(\mu_{C}^{B}-\mu_{O}^{B}\right)
$$

the formation energy in Eq. 6 can be rewritten as

$$
\Omega_{D}=E_{D}^{\prime}-\frac{1}{4}\left(n_{C}+n_{O}-n_{T i}\right) \Delta \bar{\mu}-\frac{1}{2}\left(n_{C}-n_{O}\right) \delta \bar{\mu}
$$

where

$$
\begin{aligned}
E_{D}^{\prime}=E_{D} & -\frac{1}{2}\left(n_{C}-\frac{n_{T i}}{2}\right)\left(\mu_{C}^{B}-\mu_{T i}^{B}\right)-\frac{1}{2}\left(n_{O}-\frac{n_{T i}}{2}\right)\left(\mu_{O}^{B}-\mu_{T i}^{B}\right) \\
& -\frac{1}{4}\left(n_{C}+n_{O}+n_{T i}\right) \mu_{T i C_{0.5} O_{0.5}}^{B}-\frac{1}{4}\left(n_{C}-n_{O}\right)\left(\mu_{C}^{B}-\mu_{O}^{B}\right)
\end{aligned}
$$

which only depends of the bulk chemical potencial of Ti, C and O. On the other hand, the formation energy $\Omega{ }_{D}$ only depends on $\Delta \bar{\mu}$ and $\delta \bar{\mu}$. In the case of the ternary compound, we have

$$
-\Delta \bar{H} \leqslant \Delta \bar{\mu} \leqslant \Delta \bar{H}
$$




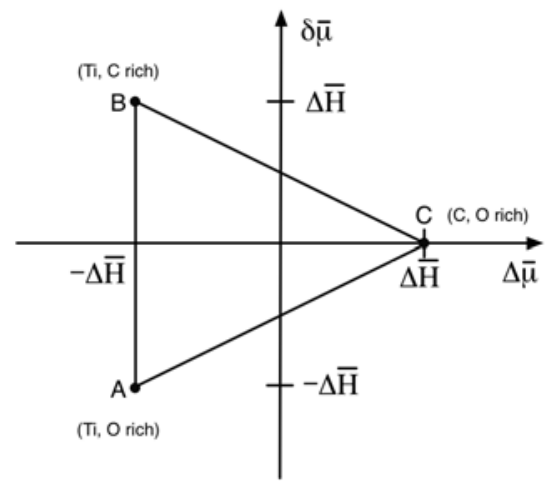

Figure 2: The Ti- $(\mathrm{A} \rightarrow \mathrm{B}), \mathrm{C}-(\mathrm{B} \rightarrow \mathrm{C})$ and $\mathrm{O}$-rich $(\mathrm{C} \rightarrow \mathrm{A})$ limits are represented as function of $\Delta \mu$ and $\delta \mu$, which change along the range bounded by the triangle.

where

$$
\Delta \bar{H}=\left(\mu_{O}^{B}+\mu_{C}^{B}+2 \mu_{T i}^{B}\right)-\mu_{T i C_{0.5} O_{0.5}}^{B}
$$

The parameter $\delta \bar{\mu}$ is restricted by the constraint

$$
-\frac{1}{2}(\Delta \bar{H}-\Delta \bar{\mu}) \leqslant \delta \bar{\mu} \leqslant \frac{1}{2}(\Delta \bar{H}-\Delta \bar{\mu})
$$

In the Ti-rich condition $(\Delta \bar{\mu}=-\Delta \bar{H}), \delta \bar{\mu}$ varies from $-\Delta \bar{H}$ (O-rich limit) to $\Delta \bar{H}$ (C-rich limit). In O- and C- rich conditions $(\Delta \bar{\mu}=\Delta \bar{H})$ thus $\delta \bar{\mu}$ is equal to zero. Figure 2 shows the different chemical environments defined by $\Delta \bar{\mu}$ and $\delta \bar{\mu}$. Note that $\Delta \bar{\mu}$ has a minimum value at Ti-rich conditions and a maximum at Ti-poor conditions.

The formalism presented in this section, for binary and ternary alloys, was used to predict the relative occurrence probability of several types of structural single point defects in $\mathrm{TiC}$, $\mathrm{TiO}$ and $\mathrm{TiC}_{0.5} \mathrm{O}_{0.5}$ compounds for different chemical growth conditions.

\section{Results and Discussion}

\subsection{Vacancies and antisites in $\mathrm{TiC}$ and $\mathrm{TiO}$}

Table 1 presents the vacancy $\left(\mathrm{V}_{T i}\right.$ and $\left.\mathrm{V}_{C}\right)$ and antisite $\left(\mathrm{Ti}_{C}\right.$ and $\left.\mathrm{C}_{T i}\right)$ formation energies for TiC compound. The formation energies of all defects are also plotted in figure 3 for the different equilibrium conditions.

The $\mathrm{V}_{C}$ is found to be the most probable defect in $\mathrm{TiC}$, which is in agreement with experiments [4]. In Ti-rich environment the $\mathrm{V}_{C}$ has a negative formation energy meaning that under this condition carbon vacancies are created spontaneously. However, this may happen only when enough thermal energy is available to surmount an eventual potential barrier. The other defects are very unlikely to occur because their formation requires high energies.

The calculated formation energies of vacancies $\left(\mathrm{V}_{T i}, \mathrm{~V}_{O}\right)$ and antisite $\left(\mathrm{Ti}_{O}, \mathrm{O}_{T i}\right)$ defects for $\mathrm{TiO}$ are presented in the table 2 and plotted in Figure 4 for the different chemical environments. 


\begin{tabular}{ccccc}
\hline \hline & $\mathrm{V}_{T i}$ & $\mathrm{~V}_{C}$ & $\mathrm{Ti}_{C}$ & $\mathrm{C}_{T i}$ \\
\hline Ti-rich & 9.34 & -0.95 & 5.88 & 14.90 \\
C-rich & 7.49 & 0.89 & 9.58 & 11.20 \\
\hline \hline
\end{tabular}

Table 1: Formation energies $\left(\Omega_{D}\right)$ in $\mathrm{eV}$, of vacancies $\left(\mathrm{V}_{T i}, \mathrm{~V}_{C}\right)$ and antisites $\left(\mathrm{Ti}_{C}, \mathrm{C}_{T i}\right)$ in TiC.

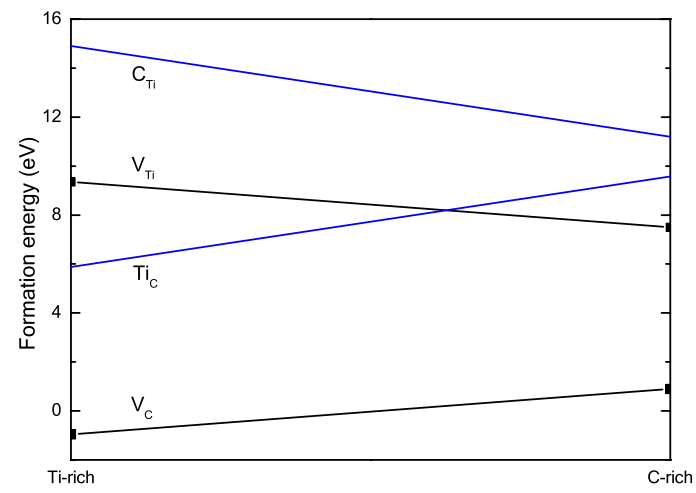

Figure 3: Defect formation energies $\left(\Omega_{D}\right)$ as function of chemical potentials from Ti-rich limit to the C-rich limit, for TiC.

\begin{tabular}{ccccc}
\hline \hline & $\mathrm{V}_{T i}$ & $\mathrm{~V}_{O}$ & $\mathrm{Ti}_{O}$ & $\mathrm{O}_{T i}$ \\
\hline Ti-rich & -0.74 & -1.37 & 6.74 & 7.75 \\
O-rich & -6.82 & 4.71 & 18.91 & -4.41 \\
\hline \hline
\end{tabular}

Table 2: Formation energies $\left(\Omega_{D}\right)$ in $\mathrm{eV}$, of vacancies $\left(\mathrm{V}_{T i}, \mathrm{~V}_{O}\right)$ and antisites $\left(\mathrm{Ti}_{O}, \mathrm{O}_{T i}\right)$ in $\mathrm{TiO}$.

Under Ti-rich conditions the $\mathrm{V}_{T i}$ and $\mathrm{V}_{O}$ formation energies are both negative and are highly probable to occur with formation energy of -0.74 and -1.37 respectively. This results are supported by the experimental observation of vacancies in both lattices of $\mathrm{TiO}$ compounds $[5,14]$. The antisites are unlikely to occur in Ti-rich conditions due to their high formation energies. Oxidation conditions are favorable to the formation of $\mathrm{O}$ antisites $\left(\mathrm{O}_{T i}\right)$ and Ti vacancy defects $\left(\mathrm{V}_{T i}\right)$ in the metallic lattice, due to their negative formation energies. This suggests an imminent phase transition towards a substoichiometric $\mathrm{TiO}_{2}$. The relatively high formation energy of $\mathrm{V}_{O}$ and $\mathrm{Ti}_{O}$ defects means that under O-rich conditions they are hard to be formed in $\mathrm{TiO}$.

\subsection{Vacancies in $\mathrm{TiC}_{0.5} \mathrm{O}_{0.5}$}

The formation energy of vacancies was studied for the ternary compound $\mathrm{TiC}_{0.5} \mathrm{O}_{0.5}$ as function of the chemical potential difference (see figure 2). Table 3 presents values obtained for the formation energies of $\mathrm{Ti}, \mathrm{C}$ and $\mathrm{O}$ vacancies 


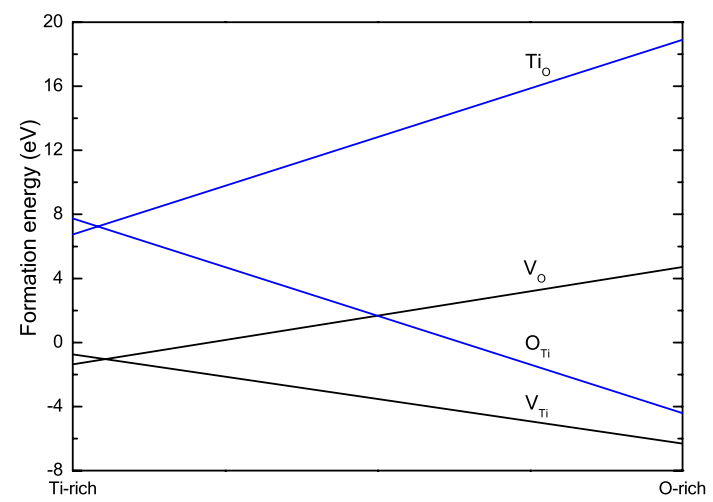

Figure 4: Defect formation energies as function of atomic chemical potentials between two extremes, the Ti-rich and O-rich conditions, for TiO.

$\left(\mathrm{V}_{T i}, \mathrm{~V}_{C}\right.$ and $\left.\mathrm{V}_{O}\right)$ in $\mathrm{TiC}_{0.5} \mathrm{O}_{0.5}$, for the different chemical environments.

\begin{tabular}{cccc}
\hline \hline & Ti-, O-rich & Ti-, C-rich & C-, O-rich \\
\hline $\mathrm{V}_{T i}$ & 2.40 & 2.40 & -1.80 \\
$\mathrm{~V}_{C}$ & -7.59 & 0.82 & -7.59 \\
$\mathrm{~V}_{O}$ & 6.10 & -2.31 & 6.10 \\
\hline & C-poor & O-poor & Ti-poor \\
\hline \hline
\end{tabular}

Table 3: Formation energies $\left(\Omega_{D}\right)$ of vacancies $\left(\mathrm{V}_{T i}, \mathrm{~V}_{C}\right.$ and $\left.\mathrm{V}_{O}\right)$ for $\mathrm{TiC}_{0.5} \mathrm{O}_{0.5}$

In Ti-rich and C-poor conditions $(\mathrm{A})$ the carbon vacancy $\left(\mathrm{V}_{C}\right)$ is highly probable to occur. In this conditions the other defects, $\left(\mathrm{V}_{T i}\right.$ and $\left.\mathrm{V}_{O}\right)$ hardly occur due to their high formation energies. In C-rich and O-poor conditions (B), $\mathrm{V}_{O}$ has the lowest formation energy and $\mathrm{V}_{T i}$ has the highest one. These are the unique conditions favorable to the occurrence of $\mathrm{V}_{O}$ defect in $\mathrm{TiC}_{0.5} \mathrm{O}_{0.5}$. For O-rich and Ti-poor conditions (C), $\mathrm{C}$ and Ti vacancies are likely to occur. As expected, in O-rich atmospheres the occurrence of $\mathrm{O}$ vacancies are unlikely to occur.

\section{Conclusions}

This work provides a general overview of the formation of several types of structural point defects in titanium oxycarbides for different chemical growth conditions. We used first principles methods to calculate the formation energies of vacancies and antisite defects in $\mathrm{TiC}, \mathrm{TiO}$ compounds and the formation energy of vacancies in a ordered TiCO alloy with composition $\mathrm{TiC}_{0.5} \mathrm{O}_{0.5}$. Our calculations suggest that $\mathrm{C}$ vacancies are likely to be formed during the growth of $\mathrm{TiC}$ and $\mathrm{TiC}_{0.5} \mathrm{O}_{0.5}$, whereas $\mathrm{Ti}$ vacancies are highly probable to occur during the growth of $\mathrm{TiO}$ and $\mathrm{O}$ 


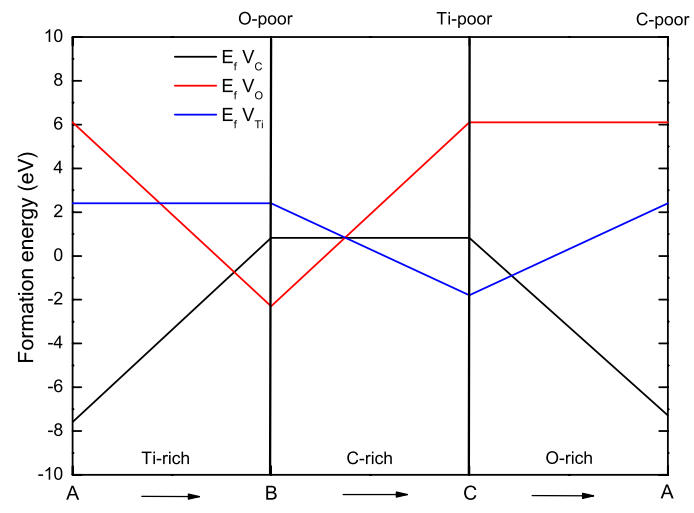

Figure 5: The defect formation energies are plotted in the Ti-, C-, and O-rich limits for $\mathrm{TiC}_{0.5} \mathrm{O}_{0.5}$.

antisite defects are also likely to be formed on O-rich atmospheres leading to an imminent phase transition towards a substoichiometric $\mathrm{TiO}_{2}$. The obtained results are supported by the existent experimental studies of point defects in $\mathrm{TiC}$ and $\mathrm{TiO}$ compounds.

\section{References}

[1] J. Yao, J. Shao, H. He, Z. Fan, Vacuum 2007, 8, 1023.

[2] A.T. Santhenam, Application of transition metal carbides and nitrides in industrial tools, in: S.T. Oyama (Ed.), The Chemistry of Transition Metal Carbides and Nitrides, Blackie Academic and Professional, London 1996, 28.

[3] A. R. Bally, P. Hones, R. Sanjins, P. E. Schmid, F. Lvy, Surface and Coatings Technology 1998, 108, 166.

[4] M. Guemmaz, A. Mosser, J. C. Parlebas, J Electr Spectr Rel Phenom 2000, 107, 91.

[5] A. A. Valeeva, A. A. Rempel, W. Sprengel, H. E. Schaefer, Phys. Rev. B 2007, 75, 94107.

[6] P. Hohenberg, W. Kohn, Phys. Rev. B 1964, 136, 864.

[7] G. Kresse and J. Furthmüller, Comput. Math. Sci. 1996, 6, 15.

[8] G. Kresse and J. Furthmüller, Phys. Rev. 1996, 54, 11169.

[9] G. Kresse and J. Hafner, Phys. Rev. B 1997, 47, 558.

[10] P.E. Blöchl, Phys. Rev. B 1994, 17, 953.

[11] J. H. Monkhorst, J. Pack, Phys. Rev. 1976, 13, 5188.

[12] S. B. Zhang and John E. Northrup, Phys. Rev. B 1991, 47, 6791.

[13] Sun-Ghill Lee and K. J. Chang, Phys. Rev. B 1996, 53, 9785.

[14] A. A. Valeeva, A. A. Rempel, and A. I. Gusev, Inorg. Mater. 2001 37, 603. 